\title{
Instrumental Activities of Daily Living Questionnaires for Dementia and Mild Cognitive Impairment
}

\author{
Paul Regal ${ }^{\mathrm{a}, \mathrm{c}}$, Aileen Carter ${ }^{\mathrm{b}}$
}

\begin{abstract}
Background: Our aim was to explore the role of instrumental activities of daily living (IADL) questionnaires in transition from no cognitive impairment to mild cognitive impairment (MCI) and dementia in comparison to neuropsychological tests.

Methods: We monitored 397 community-dwelling elderly from a Memory Clinic every 6 months with Nottingham IADL, mini-mental state examination (MMSE), Montreal cognitive assessment (MoCA), Addenbrooke cognitive assessment and frontal assessment battery.

Results: IADL impairment in older people free of dementia was a powerful predictor of incident dementia similar in magnitude to neuropsychological tests. IADL had high correlations $(r=0.37-0.47)$ with initial MMSE, MoCA, frontal assessment battery and Addenbrooke cognitive examination-R (ACE-R) with highly significant $\mathrm{P}$ values $(<0.0001)$. There were also high correlations between IADL at baseline and at 6, 12 and 24 months. Literature search disclosed 22 articles measuring IADL questionnaires (IADL-Q).
\end{abstract}

Conclusions: IADL-Q are a simple cost-free method for measuring cognitive function and predicting incident dementia.

Keywords: Activities; Daily living; Instrumental activities; Dementia; Mild cognitive impairment

\section{Introduction}

The American Psychiatric Association has published a series of Diagnostic and Statistical Manuals for Mental Disorders (DSM) [1] defining criteria for dementia. From DSM-III

Manuscript accepted for publication December 22, 2014

aUniversity of Newcastle, PO Box 105, Wyong, NSW 2659, Australia bWyong Hospital, PO Box 4200, Lake Haven, NSW 2663, Australia ${ }^{\mathrm{c} C}$ Corresponding Author: Paul Regal, University of Newcastle, PO Box 105, Wyong, NSW 2659, Australia. Email: Regalelderlymedicine@gmail.com

doi: http://dx.doi.org/10.14740/jnr316w
(1980) to DSM-V (2013), the criteria for diagnosis of dementia required a drop in social and occupational function. Cognitive decline without significant loss of social and occupational function is termed cognitive impairment not dementia (CIND) or mild cognitive impairment (MCI). Ronald Petersen's criteria for MCI [2] require a 1.5 SD decline in memory and/or other cognitive domains.

Despite this centrality of occupational dysfunction in the DSM-IV criteria for dementia, there is a surprising little research into defining cutoffs for activities of daily living (ADL) and instrumental activities of daily living (IADL) in dementia, MCI or subjective cognitive impairment (SCI). Likewise relatively few investigators have compared ADL and IADL to neuropsychological tests in populations with no cognitive impairment (NCI) or MCI. IADL is a more sensitive test than ADL for dementia. Monaci and Morris [3] found that at baseline the average ADL score in 34 patients with dementia was $88 \%$ of ceiling whereas the average IADL score was $61 \%$. The average change over mean 21-month follow-up was a $2.2 \%$ improvement in ADL compared to $6.4 \%$ decline in IADL. We have seen this clinically because IADL functions such as self-medication and cooking are affected early in the course of dementia whereas showering and toileting become impaired much later.

Our aim was to examine IADL in the transition from NCI to $\mathrm{MCI}$ and on to dementia. We compared the IADL-Q to the benchmark tests cited in the literature on a sample of community-dwelling elderly. At least $90 \%$ of IADL tasks are relevant to the average older person. Some will have given up driving, gardening or using a conventional oven years before the current illness.

This review will focus on IADL-Q rather than IADL measurements for four reasons. First the IADL-Q gives a retrospective view (like turning back time) provided there was a reliable informant who observed the patient at that time interval. We can easily ask the daughter of a 90-year-old to describe and rate her mother's IADL at various past intervals to plot the trajectory of IADL decline. With each decline we seek evidence of factors other than cognition to account for the change such as hip fracture, stroke or Parkinson's disease. Other tests which share this unique role include exercise tolerance, ability to play a musical instrument or a game of skill such as chess. Second IADL-Q is brief and costs virtually nothing: a single sheet of paper is the only consumable unless the test is administered by 
computer screen, which eliminates the sheet of paper. Scoring takes less than $90 \mathrm{~s}$ for the paper version and less than $5 \mathrm{~s}$ for a computer version. Third, there is no problem with multiple testing compared to asking a patient for orientation items such as the date or their home address. Forth, almost every IADL item is weighted as important by the patient and family, in contrast to copying a cube drawing in the Montreal cognitive assessment (MoCA) [4] or Addenbrooke cognitive examination (ACE-R) [5], which is relevant only to people involved in design such as sign writing or architecture.

In this article we report data as mean $\pm \mathrm{SD}$ unless the investigator providing the data found the figures skewed and reported median with quartiles. The relative changes on the 22-point Nottingham (N-IADL) scale [6] are reported as the absolute change between measurements divided by 22 . Thus a two-point absolute change represents $2 / 22(9.1 \%)$ relative change. Unless stated otherwise all IADL ratings are by questionnaire rather than by direct measurement by an occupational therapist. Direct measurement is the gold standard but is applicable only in the here and now - one cannot go back in time to measure IADL 12 months ago.

Our aim was to examine IADL in the transition from NCI to MCI and on towards dementia to determine whether IADL$\mathrm{Q}$ can be used to diagnose dementia.

\section{Method}

The Wyong Hospital is a 370-bed institution located $80 \mathrm{~km}$ north of Sydney Australia. The Wyong Memory Clinic serves patients aged 60+ years with NCI, SCI, MCI and dementia. We analyzed 397 community-dwelling memory clinic patients with mean age of $80.4 \pm 6.6$ years. Eighty-one percent of Memory Clinic subjects were community-dwelling, so this sample was drawn from 490 referrals, primarily from GPs but also from hospital units and specialists. Patients were assessed at 6-month intervals with N-IADL [6], ACE-R [5], mini-mental state examination (MMSE) [7], frontal assessment battery (FAB) [8] and MoCA [3]. PR measured MoCA and FAB while the dementia clinical nurse consultant measured MMSE and ACE-R. An absolute change of two points on the 22-point IADL scale represented a relative change of $2 / 22(9.1 \%)$. Recruitment began in January 2009 and ended in January 2013. Results were analyzed using Stats Direct version 2.7.9 2012.

\section{Results}

The mean age of 397 subjects was $80.4 \pm 6.6$ years. One hundred ninety-four $(48.9 \%)$ were female. One hundred forty-one out of $397(35.5 \%)$ lived alone. Baseline IADL was 13.7 \pm 5.5 , MMSE was $22.7 \pm 5.2$, MoCA was $17.0 \pm 6.3$, FAB was $9.7 \pm$ 4.0, and ACE-R was $66.0 \pm 17.4$. IADL at 6,12 and 24 months were $12.9 \pm 6.1,12.2 \pm 6.2$ and $10.7 \pm 6.6$. All Spearman rank correlations between initial IADL and baseline, 6-month and 12-month MMSE, MoCA, FAB, ACE-R-R and IADL were significant at $\mathrm{P}<0.0001$ level. The typical correlation coefficients between baseline IADL and IADL at 6, 12 and 24
Table 1. Serial Values of Instrumental Activities of Daily Living (IADL), MMSE, MoCA, FAB at ACE-R for All Subjects

\begin{tabular}{llllll}
\hline Months & IADL & MMSE & MoCA & FAB & ACE-R \\
\hline Baseline & 13.7 & 22.7 & 17.0 & 9.7 & 66 \\
6 & 12.9 & 22.0 & 17.0 & 10.0 & 69 \\
12 & 12.2 & 23.0 & 17.0 & 10.0 & 71 \\
18 & 10.7 & 22.0 & 16.0 & 9.0 & 68 \\
24 & 10.7 & 23.0 & 17.0 & 10.0 & 68 \\
\hline
\end{tabular}

months were between 0.75 and 0.91 , whereas the correlation coefficients between baseline IADL and the four cognitive test scores were between 0.32 and 0.47 . Serial values for the cognitive scores are shown in Table 1.

We next divided the 397 community-dwelling Memory Clinic patients into two groups: 272 who remained community-dwelling at a mean of 22.3 months and 125 who either died $(\mathrm{N}=57 / 125)$, entered low-level care (hostels $\mathrm{N}=32 / 125)$ or high-level residential care (nursing homes $\mathrm{N}=36 / 125$ ). Mean age was $79.4 \pm 6.5$ vs. $82.7 \pm 6.5$ years $(\mathrm{P}<0.0001)$; female $51.1 \%$ vs. $53.6 \%(\mathrm{P}=0.645)$; living alone at baseline $29.8 \%$ vs. $48.0 \%(\mathrm{P}=0.0006)$; baseline IADL was 3.0 point higher than the residential care group (relative change $3.0 / 22$ or $13.6 \%, \mathrm{P}<0.0001$, Table 2); baseline MMSE was 2.5 points higher (relative change $2.5 / 30$ or $8.3 \%, \mathrm{P}<0.0001$ ); baseline MoCA 4.4 points higher (relative change $4.4 / 30$ or $14.7 \%, \mathrm{P}$ $<0.0001$ ); baseline FAB 2.5 points higher (relative change $2.5 / 18$ or $13.9 \%, \mathrm{P}<0.0001$ ); baseline ACE-R-R 9.6 higher (relative change $9.6 / 100$ or $9.6 \%, \mathrm{P}<0.0001$ ); 6 -month IADL $14.5 \pm 5.5$ vs. $9.0 \pm 5.6(\mathrm{P}<0.0001)$. Thus, baseline IADL had the same predictive value as baseline MMSE, MoCA, FAB and ACE-R-R for institutionalization or death over a mean 22-month follow-up.

\section{Discussion}

\section{Literature review of IADL for diagnosis of dementia and MCI}

PR reviewed 2,590 articles in English from his article file based on multiple medical journals. This search yielded 22 articles reporting IADL published in a mean of $2011.2 \pm 1.4$ (median 2012). Clare et al [9] found a significant discrepancy between self-rated and informant-rated IADL, averaging 3540\%. Larner and Hancock [10] reported 79 patients with median age of 76 years. Fifty-seven percent had DSM-IV dementia. They compared four combinations of Lawton Brody IADL scores [11] (LB-IADL cutoff < 14/14) and ACE-R-R (cutoff < 73/100): group I had abnormal LB-IADL with normal ACE$\mathrm{R}-\mathrm{R}$, group II had abnormal ACE-R-R with normal LB-IADL, group III had abnormal LB-IADL and ACE-R (termed in series), group IV LB-IADL or ACE-R parallel: accuracy 0.78 vs. 0.82 vs. 0.80 vs. 0.81 ; sensitivity 0.91 vs. 0.76 vs. 0.69 vs. 0.98 (best sensitivity for parallel); specificity $0.62 \mathrm{vs} .0 .91 \mathrm{vs}$. 0.94 vs. 0.59 (best specificity for serial 0.94 followed close 
Table 2. Comparison of Serial Values of Instrumental Activities of Daily Living (IADL), MMSE, MoCA, FAB at ACE-R in Community-Dwelling Versus Residential Care or Subjects Who Died

\begin{tabular}{llllll}
\hline Group & IADL & MMSE & MoCA & FAB & ACE-R \\
\hline Community & 14.0 & 23.5 & 18.5 & 10.5 & 68.1 \\
Residential care or died & 11.0 & 21.0 & 14.1 & 8.0 & 58.5 \\
\hline
\end{tabular}

second by ACE-R); positive predictive value 0.76 vs. 0.92 vs. 0.94 vs. 0.76 (best PPV for serial 0.94 followed closely by ACE-R 0.92); negative predictive value 0.84 vs. 0.74 vs. 0.70 vs. 0.95 (best NPV for parallel). This study is unique in comparing parallel and serial IADL with a neuropsychological test (ACE-R) and established IADL as a robust predictor of incident dementia.

Hancock and Larner [12] recruited 296 referrals to Memory Clinic in Liverpool. Fifty-two percent had dementia. They used a four-item modified LB-IADL which was not sensitive to diagnose dementia.

\section{IADL in longitudinal studies}

Luck et al [13] followed LB-IADL over a mean of 4.5 years in the German Study on Ageing, Cognition and Dementia in Primary Care Patients (AgeCoDe). They recruited an enormous sample of 3,327 patients from 138 GPs. Patients were assessed every 1.5 years over 4.5 years. The authors used different LB-IADL cutoffs for women and men: norm for women was $8 / 8$ compared to $5 / 5$ in men (they excluded food preparation, house cleaning and laundry for men). They used the structured interview for diagnosis of Alzheimer disease (SIDAM) which had two components: 1) cognitive test with 55 items, of which 30 items (54\%) come from MMSE [7]; 2) ADL/clinical judgment. SIDAM-ADL has 14 items. Nine point seven percent had impaired LB-IADL at baseline. Incident dementia occurred in $10.6 \%$ of 3,198 patients over 4.5 years. They placed patients into two broad categories of cognition: NCI or MCI. Over 4.5 years mean follow-up, $10.6 \%$ converted to dementia. Conversion rate was lowest in the group with NCI and normal IADL (7.1\%), $19.6 \%$ in the group with MCI but normal IADL, $20.2 \%$ in those with NCI and impaired IADL and 50.0\% in the group with MCI plus impaired IADL at baseline. The group with impaired IADL and normal or impaired baseline cognition had a $27.1 \%$ conversion rate to dementia compared to $8.8 \%$ conversion for those with normal IADL at baseline. Given the fact that the average study of its kind has 250 subjects this investigation is 13.3-fold larger. Germans with impaired IADL have a higher conversion to dementia.

Luck et al [14] studied 819 subjects free of dementia at baseline in the Leipzig Longitudinal Study of the Aged (LEILA75+) from a population sample of age $75+$ communitydwelling plus 192 randomly sampled residents in four institutions. They conducted five visits every 1.4 years. MCI was diagnosed by Winblad et al criteria of 2004 [15] rather than by the Petersen criteria (1999). During 7-year follow-up 22.0\% developed dementia. The group with impaired IADL at baseline had $31.2 \%$ conversion to dementia compared to $11.1 \%$ conversion for those with normal baseline IADL $(\mathrm{P}<0.001)$. Conversion of subjects with MCI at baseline $(41.9 \%)$ was higher than in those with NCI at baseline $(17.6 \%, \mathrm{P}<0.001)$. Conversion of MCI with impaired IADL at baseline $(47.4 \%)$ compared to MCI with normal IADL (31.4\%) compared to $\mathrm{NCI}+$ impaired IADL $(26.7 \%)$ vs. NCI with normal IADL $(8.0 \%)(\mathrm{P}<0.001)$. Thus from best to worst: 1$) \mathrm{NCI}$ with normal IADL had an $8.0 \%$ conversion to dementia; 2) NCI with impaired IADL had a $26.7 \%$ conversion to dementia; 3) MCI with normal IADL had a $31.4 \%$ conversion to dementia; 4) MCI with impaired IADL had a $47.4 \%$ conversion to dementia. Thus IADL predicted later dementia.

Boyle et al [16] followed 761 elderly in Chicago free of MCI and dementia at baseline for mean 6.0 years. They conducted annual assessments which included Katz ADL, OARS IADL and 21 neuropsychological tests. Forty point one percent developed incident MCI. Comparing $456 \mathrm{NCI}$ at followup with $305(40 \%)$ incident MCI, the number of baseline OARS IADL disabilities was 0.6 vs. $1.0(\mathrm{P}<0.001)$ and the percentage with at least one IADL disability 12.2 vs. $25.6(\mathrm{P}$ $<0.001)$. After controlling for baseline disability, the hazard ratios (HRs) for incident MCI were 1.46 for IADL and 1.59 for ADL. Hence impaired ADL was slightly more predictive of MCI than IADL.

Folstein et al [7] assessed 101 Welch patients with early dementia. The functional activities questionnaire (FAQ) [17] has 10 IADL items each scored 0 (independent) to 3 (totally dependent). Self-rated IADL worsened over time from $6.18 \pm$ 5.46 at baseline to $8.84 \pm 5.91$ at 12 months (absolute difference 2.88 , relative change $2.88 / 30=9.6 \% ; 10.03 \pm 7.04$ at 20 months (absolute change from baseline 3.85 , relative change $3.85 / 30=12.8 \%$ ). Informant-rated IADL worsened over time: FAQ $16.4 \pm 8.3$ at baseline; $20.8 \pm 8.14, \mathrm{n}=66$ at 12 months (absolute change 4.44, relative change $4.44 / 30=14.8 \%$ ); 21.6 $\pm 8.16, \mathrm{n}=51$ at 20 months (absolute change from month 0 5.25 , relative change $17.5 \%$ ). The difference between informant- and self-rated IADL was 10.18 at baseline on 30 point scale $(33.9 \%), 11.96 / 30(39.9 \%)$ to 12 months, and 11.61/30 (38.7\%) to 20 months. Neither was compared to actual IADL.

Farias et al [18] studied 100 elderly with the eight-item BR-IADL from Blessed-Roth dementia rating scale [19]. Each item was rated 0 (normal performance/no difficulty), 0.5 (some difficulty), and 1 (unable to perform). Total score ranged from 0 (best) to 8 (worst). They conducted 483 BR-IADL assessments on average of 1 year apart. Comparing 45 NCI with 29 MCI and 26 dementia patients baseline BR-IADL was $0.28 \pm$ 0.69 vs. $0.65 \pm 0.70$ vs. $2.6 \pm 1.9$; annual percentage change was $0.09(0.09 / 8=0.011 \%)$ vs. $0.21(0.21 / 8=0.026 \%)$ vs. 0.8 $(0.8 / 8=0.10 \%)$. Thus annual percentage worsening in BRIADL was most rapid in dementia, intermediate in MCI and 
slowest in NCI.

Hughes et al [20] studied OARS-IADL in $623 \mathrm{MCI}$ and 1,114 NCI aged $65+$ years community-dwelling subjects with mean age of 77.2 years. Comparing 1,114 NCI to $623(35.9 \%)$ with MCI, dependency on OARS-IADL (scores 1 - 7) was $10.1 \%$ vs. $18.6 \%(\mathrm{P}<0.0001)$.

Jefferson et al [21] studied 77 community-dwelling subjects free from dementia. Thirty-eight had MCI and 39 NCI. Caregivers rated IADL-PSMS (which included telephone use, housekeeping, laundry, shopping, meal preparation, and medication management). Comparing NCI to MCI, IADL was 15.7 vs. $15.1(\mathrm{P}<0.03)$. Koepsell and Monsell [22] followed 3,020 MCI patients measuring IADL by FAQ. Sixteen percent reverted from MCI to NCI, $19.7 \%$ progressed to dementia and $64.3 \%$ remained MCI. FAQ score predicted reversion of MCI to NCI and progression of MCI to dementia $(\mathrm{P}<0.001)$.

Lo and Jagust [23] reported 810 subjects divided into three groups: $229 \mathrm{NCI}, 397 \mathrm{MCI}$ and $193 \mathrm{AD}$. IADL was by 10 -item FAQ. Follow-up for AD was 2 years whereas $\mathrm{NCI}$ and $\mathrm{MCI}$ had 3-year follow-up (FAQ $0.1 \pm 0.6$ for NCI vs. $3.9 \pm 4.5$ for MCI vs. $13.0 \pm 6.8$ for $\mathrm{AD}$ ).

Monaci and Morris [3] studied 34 community-dwelling London patients with mild $\mathrm{AD}$ (MMSE $\geq 21$ ) or moderate $\mathrm{AD}$ (MMSE 10 - 20). They administered: 1) CAMCOG-R [24] scored from 0 to $105 ; 2$ ) MMSE; 3) executive function scale [23] scored $0-28$, ideational fluency, visual reasoning, word list fluency, and similarities. Family rated ADL and LB-IADL. Total modified IADL score 0 (worst) to 14 (best). Katz ADL was scored 0 - 12. Comparing baseline to follow-up (18 - 24 months) CAMCOG-R 60.4 vs. 53.9 ( $\mathrm{P}<0.0001)$; MMSE 19.6 vs. $18.1(\mathrm{P}<0.05)$; EFS 12.0 vs. $10.5(\mathrm{P}<0.05)$; Katz 10.6 vs. $10.9(\mathrm{P}=0.98)$; modified IADL ( 0 - 14) 8.55 vs. 7.65 at 18 $24 / 12(\mathrm{P}=0.109)$. Baseline IADL was significantly correlated with baseline MMSE $(r=0.73, P<0.0001)$, CAMCOG-R $(r=$ $0.76, \mathrm{P}<0.0001)$, and EFS $(\mathrm{r}=0.73, \mathrm{P}<0.0001)$. IADL at 18 24 months was not correlated with follow-up MMSE $(r=0.27$, $\mathrm{P}=0.140)$, CAMCOG-R $(\mathrm{r}=0.27, \mathrm{P}=0.139)$, EFS $(\mathrm{r}=0.17$, $\mathrm{P}=0.356)$. Unlike IADL, Katz ADL at baseline or follow-up was not correlated with MMSE, CAMCOG-R or EFS.

Reppermund et al [25] reported on 762 community-dwelling subjects. Exclusions were dementia and major neuropsychological disorders. They used the Bayer ADL 0 (no difficulties) to 10 (always having difficulties). They summed the 25 Bayer items and divided by 10 to give B-ADL from 1 (best) to 10 (worst). They defined MCI by the Petersen criteria. They conducted factor analysis of B-ADL: 1) factor 1 high cognitive demand: coping with unfamiliar situations, performing a task under pressure, describing what he or she has just seen, continuing the same task after a brief interruption, taking a message for someone else, observing important dates or events, and doing two things at the same time; 2) factor II low cognitive demand: using transportation, shopping, taking a walk without getting lost, taking care of yourself, managing everyday activities, preparing food, personal hygiene, and using home appliances. The 293 who converted from NCI to MCI had a significantly different Bayer ADL at baseline: $1.56 \pm 0.58$ vs. $1.39 \pm 0.51(\mathrm{P}<0.001)$. Bayer high cognitive demand differed significantly between converters and non-converters but not
Bayer low cognitive demand.

Reppermund [26] reported additional IADL data. Thirtythree percent of MCI patients converting to dementia have impaired IADL compared to $12 \%$ of MCI non-converters. Predictors of conversion from NCI to MCI at 2 years included Bayer-ADL high cognitive demand (HR 1.54, $\mathrm{P}<0.05)$ but not Bayer low cognitive demand (HR 0.84). Predictors of conversion from NCI to dementia at 2 years included Bayer ADL high cognitive demand (HR 3.55, $\mathrm{P}<0.01$ ).

Sarker et al [27] assessed 238 stroke patients in London surviving 3 months using N-IADL, Frenchay activities index (FAI), and Barthel ADL. Pre-stroke Barthel was 20/20 in $80.8 \%$. At 3 months floor effect was in $19 \%$ of FAI vs. $4 \%$ IADL vs. $2 \%$ Barthel $(\mathrm{P}<0.001)$. At 3 months ceiling effect was in 33\% Barthel, $4 \%$ IADL and $0 \%$ FAI $(\mathrm{P}<0.001)$. Spearman rank correlations were IADL vs. FAI $(r=0.90)$, IADL vs. BI $(r=0.88)$, FAI vs. Barthel $(r=0.80)$.

Sikkes et al [28] followed 531 patients free from dementia at baseline for 12 months and 481 for 24 months. Dementia was defined by DSM-IV criteria. Only 16/20 clinics (80\%) measured IADL. LB-IADL was the most common scale (56.2\%). Three out of 16 centers used the Blessed, 1/16 the AD cooperative atudy scale and 1/16 the Bristol IADL. Thirteen percent developed dementia at 12 months and $20.8 \%$ at 2 years. Comparing 69 incident dementia at 12 months to 462 no dementia, baseline MMSE was 27 vs. 28 ( $\mathrm{P}<0.001)$, and memory $\mathrm{Z}$ score was -2.04 vs. $-0.94(\mathrm{P}<0.001)$. They pooled nine IADL items: cleaning, finances, food and drink preparation, handling money, laundry, medication, shopping, telephone, and transport. Subjects with at least one of nine IADL limitations at baseline had a higher 12-month dementia incidence than those with no IADL limitation: $24.4 \%$ vs. $16.7 \%(\mathrm{P}=0.04)$.

Van Kan et al [29] followed 647 community-dwelling women aged $75+$ years free of dementia and MCI for a mean of 7 years. Diagnosis of dementia was made by two experts using DSM-IV. Sixty-nine point four percent remained free of dementia at 7 years, and $22.4 \%$ developed dementia $(3.20 \%$ per year). LB-IADL was impaired in at least one of eight elements at baseline in $9.1 \%$ dementia-free at 7 years vs. $16.6 \%$ of the incident dementia $(\mathrm{P}=0.013)$. Baseline LB-IADL $\geq 1 / 8$ predicted incident dementia $(\mathrm{OR} 2.0, \mathrm{P}=0.013)$.

\section{Five cross-sectional IADL studies}

Teng et al [30] compared 1,108 MCI and 3,036 normal controls in National Alzheimer's Coordinating Center Uniform Data Set. FAQ IADL (10 items, each 0 - 3, total score 0 best to 30 worst). FAQ was significantly worse in MCI than healthy controls.

Teng et al [31] collected data from 31 Alzheimer Disease Research Centers in USA: 1,108 MCI and $696 \mathrm{AD}$ aged 50+ years with $\mathrm{MMSE} \geq 24$. Comparing the $1,108 \mathrm{MCI}$ subjects to the $696 \mathrm{AD}$ subjects, mean age was 75.8 vs. $76.8(\mathrm{P}<0.05)$; MMSE was 27.7 vs. $26.1(\mathrm{P}<0.01)$; complete FAQ IADL was $70.8 \%$ vs. $57.9 \%(\mathrm{P}<0.01)$. FAQ items that best distinguished $\mathrm{MCI}$ from $\mathrm{AD}$ were bill paying, shopping, playing games and traveling out of neighborhood. 
Teng et al [32] studied 205 elderly from Los Angeles: 97 NCI and 108 MCI. Mean age was 70.1 vs. $72.0(\mathrm{P}=0.13)$; MMSE was 28.8 vs. 27.1 ( $\mathrm{P}<0.001 \mathrm{MMSE})$; FAQ IADL was $0.06 \pm 0.24$ vs. $0.16 \pm 0.24(\mathrm{P}=0.002)$.

Delbaere et al [33] measured Bayer ADL in 419 community-dwelling subjects aged 70 - 90 free of dementia. Bayer ADL includes 24 IADL items (96\%) and one ADL item (4\%). Four out of $419(1.0 \%)$ had impaired IADL (B-ADL $\geq 3.0)-4 / 342$ NCI $(1.2 \%)$ vs. $0 / 77 \mathrm{MCI}(0 \%)$. Although this was opposite to expectation, the magnitude of difference between groups $(1.2 \%)$ was very low.

Terada et al [34] studied 40 elderly Japanese with mild AD. Patients were divided by perseverative error groups in Wisconsin card sort test (WCST). Comparing 20 with low errors to 20 with high errors, mean ACE-R-R was 68.3 vs. 68.3 $(\mathrm{P}=0.999)$; MMSE was 23.2 vs. $23.0(\mathrm{P}=0.81)$; FAB [34] was 12.0 vs. $10.9(\mathrm{P}=0.23)$; LB-IADL was 4.9 vs. $4.9(\mathrm{P}=$ 1.0). It is likely that the IADL scores of the two groups were identical because only mild AD was studied.

\section{Outcome models using IADL}

Wolfs et al [35] reviewed 349 articles to yield 10 studies of informal care in dementia. IADL was an independent variable in six of the 10 studies - it stayed in final outcome model in 6/6 $(100 \%)$ ! BPSD was assessed in $6 / 10$ studies (same as IADL) and was retained in final model of $5 / 6(83.3 \%)$ ! IADL predicted hours and costs of informal care in 5/7 (71.4\%) studies.

\section{Reasons to utilize IADL-Q for diagnosis and monitoring of MCI and dementia}

We believe that IADL is an ideal outcome measure for patients with MCI and dementia, which also serves as a predictor for later cognitive decline. IADL such as driving and cooking are highly important to patients and their families. A major decline in IADL has at least four adverse effects: 1) safety - patients may consume food that is no longer safe or cook food inadequately; 2) increased health care costs for home support services such as shopping and meal preparation; 3) carer stress; 4) ultimately lead to assisted care, supported accommodation or nursing home placement. In the Wyong Memory Clinic family or informants complete the N-IADL on each visit - this takes an average of 2 min for family and scoring takes $20 \mathrm{~s}$. The only consumable is a single sheet of paper, otherwise the test is cost-free. We therefore consider this test to be cost-free. Monaci and Morris [3] showed that baseline LB-IADL was significantly correlated with baseline MMSE ( $\mathrm{r}=0.73, \mathrm{P}<$ $0.0001)$, CAMCOG-R ( $\mathrm{r}=0.76, \mathrm{P}<0.0001)$, executive function scale $(\mathrm{r}=0.73, \mathrm{P}<0.0001)$, making it a valid tool of cognitive function. There are three limitations of this study. First we did not search occupational therapy journals. Second the memory clinic sample was from one institution. Third memory clinic has selective follow-up with drop outs after nursing home placement.

We recommend further studies comparing how different informants rate IADL-Q and comparing IADL-Q to IADL performance with an occupational therapist.

\section{Conclusions}

Medical research into dementia and MCI has focused on expensive high-technology solutions such as PIB-PET scans rather than simple non-invasive important measures such as cognitive function by IADL. Occupational therapy can provide information that cannot be duplicated by the sophisticated technology and is less expensive. Consider the hypothetical example of an 87-year-old male recluse living alone. He rarely attends his primary care physician and has never had cognitive screening let alone a full neuropsychological assessment. His son visits him once a month and gave a clear picture of normal IADL 18 months ago involving driving within a 15-min radius of home, buying groceries, preparing meals, house cleaning, managing bank accounts, listening to CD music, using a television with a remote control switch, using a washing machine and clothes drier. Fourteen months ago, he had trouble balancing bank statements. Twelve months ago, he contracted his range of cooking. Eight months ago, he started to accumulate dirty clothing. The trajectory of IADL decline warrants investigation from a physician and occupational therapist. A systematic review of articles would have generated more papers than we found but at the expense of finding many studies over 10 years old, in which the standards for diagnosing MCI may have differed from those in 2013. Basch et al [36] reported on the growing importance of patient or carer reported outcome measures such as the patient health questionnaire- 9 for monitoring recovery from depression. Their article fails to mention IADL-Q.

\section{Acknowledgement}

We thank the medical librarians of Wyong Hospital for their tireless work searching for articles on delirium and dementia: Judy Warren-Smith and Jenny Delbridge. We also thank the multi-disciplinary team of the Wyong Hospital acute care for the elderly unit.

\section{Conflict of Interest}

We declare no conflict of interest with employment, affiliations, grants, funding, honoraria, speaker's forum, consultancy, stocks, royalties, expert testimony, board membership, patents or personal relationships. We are responsible for the study concept, study design, acquisition of subjects, data management, analysis, interpretation and preparation of the manuscript. There was no sponsor.

\section{Author Contributions}

Both authors collaborated on composing the article. Paul Re- 
gal performed $90 \%$ of literature search and $100 \%$ of statistical analyses.

\section{References}

1. American Psychiatric Association. Diagnostic and Statistical Manual of Mental Disorders, 5th Edition, 2013. Washington, DC.

2. Petersen RC, Smith GE, Waring SC, Ivnik RJ, Tangalos EG, Kokmen E. Mild cognitive impairment: clinical characterization and outcome. Arch Neurol. 1999;56(3):303308.

3. Monaci L, Morris RG. Neuropsychological screening performance and the association with activities of daily living and instrumental activities of daily living in dementia: baseline and 18- to 24-month follow-up. Int J Geriatr Psychiatry. 2012;27(2):197-204.

4. Nasreddine ZS, Phillips NA, Bedirian V, Charbonneau S, Whitehead V, Collin I, Cummings JL, et al. The Montreal Cognitive Assessment, MoCA: a brief screening tool for mild cognitive impairment. J Am Geriatr Soc. 2005;53(4):695-699.

5. Mioshi E, Dawson K, Mitchell J, Arnold R, Hodges JR. The Addenbrooke's Cognitive Examination Revised (ACE-R): a brief cognitive test battery for dementia screening. Int J Geriatr Psychiatry. 2006;21(11):10781085.

6. Nouri FM, Lincoln NB. An extended activities of daily living scale for stroke patients. Clinical Rehabilitation. 1987;1:301-305.

7. Folstein MF, Folstein SE, McHugh PR. "Mini-mental state". A practical method for grading the cognitive state of patients for the clinician. J Psychiatr Res. 1975;12(3):189-198.

8. Dubois B, Slachevsky A, Litvan I, Pillon B. The FAB: a Frontal Assessment Battery at bedside. Neurology. 2000;55(11):1621-1626.

9. Clare L, Nelis SM, Martyr A, Whitaker CJ, Markova IS, Roth I, Woods RT, et al. Longitudinal trajectories of awareness in early-stage dementia. Alzheimer Dis Assoc Disord. 2012;26(2):140-147.

10. Larner AJ, Hancock P. Does combining cognitive and functional scales facilitate the diagnosis of dementia? Int J Geriatr Psychiatry. 2012;27(5):547-548.

11. Lawton MP, Brody EM. Assessment of older people: selfmaintaining and instrumental activities of daily living. Gerontologist. 1969;9(3):179-186.

12. Hancock P, Larner AJ. The diagnosis of dementia: diagnostic accuracy of an instrument measuring activities of daily living in a clinic-based population. Dement Geriatr Cogn Disord. 2007;23(3):133-139.

13. Luck T, Luppa M, Wiese B, Maier W, van den Bussche H, Eisele M, Jessen F, et al. Prediction of incident dementia: impact of impairment in instrumental activities of daily living and mild cognitive impairment-results from the German study on ageing, cognition, and dementia in primary care patients. Am J Geriatr Psychiatry.
2012;20(11):943-954.

14. Luck T, Luppa M, Angermeyer MC, Villringer A, Konig $\mathrm{HH}$, Riedel-Heller SG. Impact of impairment in instrumental activities of daily living and mild cognitive impairment on time to incident dementia: results of the Leipzig Longitudinal Study of the Aged. Psychol Med. 2011;41(5):1087-1097.

15. Winblad B, Palmer K, Kivipelto M, Jelic V, Fratiglioni L, Wahlund LO, Nordberg A, et al. Mild cognitive impairment--beyond controversies, towards a consensus: report of the International Working Group on Mild Cognitive Impairment. J Intern Med. 2004;256(3):240-246.

16. Boyle PA, Buchman AS, Wilson RS, Leurgans SE, Bennett DA. Physical frailty is associated with incident mild cognitive impairment in community-based older persons. J Am Geriatr Soc. 2010;58(2):248-255.

17. Pfeffer RI, Kurosaki TT, Harrah CH, Jr., Chance JM, Filos S. Measurement of functional activities in older adults in the community. J Gerontol. 1982;37(3):323-329.

18. Tomaszewski Farias S, Cahn-Weiner DA, Harvey DJ, Reed BR, Mungas D, Kramer JH, Chui H. Longitudinal changes in memory and executive functioning are associated with longitudinal change in instrumental activities of daily living in older adults. Clin Neuropsychol. 2009;23(3):446-461.

19. Blessed G, Tomlinson BE, Roth M. The association between quantitative measures of dementia and of senile change in the cerebral grey matter of elderly subjects. $\mathrm{Br}$ J Psychiatry. 1968;114(512):797-811.

20. Hughes TF, Chang CC, Bilt JV, Snitz BE, Ganguli M. Mild cognitive deficits and everyday functioning among older adults in the community: the Monongahela-Youghiogheny Healthy Aging Team study. Am J Geriatr Psychiatry. 2012;20(10):836-844.

21. Jefferson AL, Byerly LK, Vanderbilt S et al. Characteristics of activities of daily living in individuals with mild cognitive impairment. Am J Ger Psych. 2008;16:373383 .

22. Koepsell TD, Monsell SE. Reversion from mild cognitive impairment to normal or near-normal cognition: risk factors and prognosis. Neurology. 2012;79(15):1591-1598.

23. Lo RY, Jagust WJ. Predicting missing biomarker data in a longitudinal study of Alzheimer disease. Neurology. 2012;78(18):1376-1382.

24. Heinik J, Solomesh I. Validity of the Cambridge Cognitive Examination-Revised new Executive Function Scores in the diagnosis of dementia: some early findings. J Geriatr Psychiatry Neurol. 2007;20(1):22-28.

25. Reppermund S, Sachdev PS, Crawford J, Kochan NA, Slavin MJ, Kang K, Trollor JN, et al. The relationship of neuropsychological function to instrumental activities of daily living in mild cognitive impairment. Int $\mathrm{J}$ Geriatr Psychiatry. 2011;26(8):843-852.

26. Reppermund, Simone University of New South Wales Centre for Healthy Brain Ageing. Functional performance and mild cognitive impairment. Findings from the Sydney Memory and Ageing Study. 2012 Lecture notes.

27. Sarker SJ, Rudd AG, Douiri A, Wolfe CD. Comparison of 
2 extended activities of daily living scales with the Barthel Index and predictors of their outcomes: cohort study within the South London Stroke Register (SLSR). Stroke. 2012;43(5):1362-1369.

28. Sikkes SA, Visser PJ, Knol DL, de Lange-de Klerk ES, Tsolaki M, Frisoni GB, Nobili F, et al. Do instrumental activities of daily living predict dementia at 1- and 2-year follow-up? Findings from the Development of Screening guidelines and diagnostic Criteria for Predementia Alzheimer's disease study. J Am Geriatr Soc. 2011;59(12):22732281.

29. Abellan van Kan G, Rolland Y, Gillette-Guyonnet S, Gardette V, Annweiler C, Beauchet O, Andrieu S, et al. Gait speed, body composition, and dementia. The EPIDOS-Toulouse cohort. J Gerontol A Biol Sci Med Sci. 2012;67(4):425-432.

30. Teng E, Becker BW, Woo E, Cummings JL, Lu PH. Subtle deficits in instrumental activities of daily living in subtypes of mild cognitive impairment. Dement Geriatr Cogn Disord. 2010;30(3):189-197.

31. Teng E, Becker BW, Woo E, Knopman DS, Cummings JL, Lu PH. Utility of the functional activities questionnaire for distinguishing mild cognitive impairment from very mild Alzheimer disease. Alzheimer Dis Assoc Disord. 2010;24(4):348-353.

32. Teng E, Tassniyom K, Lu PH. Reduced quality-of-life ratings in mild cognitive impairment: analyses of subject and informant responses. Am J Geriatr Psychiatry. 2012;20(12):1016-1025.

33. Delbaere K, Kochan NA, Close JC, Menant JC, Sturnieks DL, Brodaty H, Sachdev PS, et al. Mild cognitive impairment as a predictor of falls in community-dwelling older people. Am J Geriatr Psychiatry. 2012;20(10):845-853.

34. Terada S, Sato S, Honda H, Kishimoto Y, Takeda N, Oshima E, Yokota O, et al. Perseverative errors on the Wisconsin Card Sorting Test and brain perfusion imaging in mild Alzheimer's disease. Int Psychogeriatr. 2011;23(10):1552-1559.

35. Wolfs CA, Kessels A, Severens JL, Brouwer W, de Vugt ME, Verhey FR, Dirksen CD. Predictive factors for the objective burden of informal care in people with dementia: a systematic review. Alzheimer Dis Assoc Disord. 2012;26(3):197-204.

36. Basch E, Torda P, Adams K. Standards for patient-reported outcome-based performance measures. JAMA. 2013;310(2):139-140. 